\title{
Manejo anestésico de resección de quiste de vallécula en neonato. A propósito de un caso
}

\author{
Anesthetic management of vallecular cyst resection \\ in neonate. A case report
}

Neus Fuertes S. ${ }^{1}$ MD, José Cortell B. ${ }^{2} \mathrm{MD}$, Juan Camilo Jaramillo G. ${ }^{2} \mathrm{MD}$, Pilar Argente N. ${ }^{3} \mathrm{MD}$

\begin{abstract}
Difficult airway management is one of the most important challenges an anesthesiologist faces. It is due to the high morbidity and mortality that it entails. The challenge is even greater if the patient is a newborn. For this reason, we should have different strategies that allow us to anticipate and treat possible complications derived from the procedure. In this case, we present a newborn with vallecular cyst and respiratory distress who is admitted for cyst resection. The gold-standard in anticipated difficult airway management is the fibrobronchoscope. We decided to perform an alternative management by means of orotracheal intubation with videolaryngoscope (Glydescope ${ }^{\circledR}$ ) in spontaneous ventilation.
\end{abstract}

\section{RESUMEN}

El manejo de una vía aérea difícil es uno de los retos más importantes a los que puede enfrentarse un anestesiólogo debido a la elevada morbimortalidad que conlleva. El reto aún es mayor si el paciente es un neonato. Por este motivo, debemos contar con diferentes estrategias que permitan anticipar y poder tratar las posibles complicaciones derivadas del procedimiento. En este caso, presentamos un neonato con quiste de vallécula con clínica de trabajo respiratorio que es admitido para cirugía de exéresis del quiste. El gold standard en el manejo de una vía aérea difícil conocida es el fibrobroncoscopio. Nosotros decidimos realizar un manejo anestésico alternativo mediante intubación orotraqueal con videolaringoscopio (Glydescope ${ }^{\circledR}$ ) en ventilación espontánea.

\section{Key words:}

Vallecular cyst, airway, videolaryngoscope, distress

\section{Palabras clave:}

Quiste vallécula, vía aérea, videolaringoscopio, distress

Residente de Anestesiología y Reanimación de HUiP La Fe. Valencia. España.

Adjunto de Anestesiología y Reanimación de HUiP La Fe. Valencia. España.

Jefa de Servicio de Anestesiología y Reanimación de HUiP La Fe. Valencia. España.

Fecha de ingreso: 10 de agosto de 2019

Fecha de aceptación: 02 de septiembre de 2019

\section{ORCID}

https://orcid.org/0000-0002-5221-1024

Correspondencia:

Neus Fuertes Sáez

Email: neusfuer@gmail.com 


\section{Introducción}

- I quiste de vallécula supone una causa de obs-

- trucción de vía aérea superior infrecuente en neonatos y niños, y cursa clínicamente con estridor y/o cianosis[1]. En la mayoría de los casos, puede asociar laringomalacia, que es la anomalía congénita de laringe más frecuente[2].

El manejo anestésico de estos pacientes debe realizarse con cautela, dado que el quiste de vallécula puede llegar a obstruir completamente el espacio supraglótico. En este caso, podría impedir tanto la ventilación como la intubación orotraqueal, llevando finalmente a la hipoxemia y muerte[2].

Presentamos nuestra experiencia en el manejo de una vía aérea difícil mediante videolaringoscopio (Glydescope ${ }^{\circledR}$ ) en ventilación espontánea para la exéresis de un quiste de vallécula.

\section{Método}

Recién nacida a término ( $39+3$ semanas) con peso de $3.800 \mathrm{~kg}$ y valoración escala de APGAR 9/10. Ingresa en el hospital de origen a las 2 horas de vida por quejido, precisando oxígeno a $2 \mathrm{lpm}$ mediante gafas nasales para mantener $\mathrm{SpO} 2>97 \%$. A pesar de la normalidad de las pruebas complementarias y de la buena evolución, a las 48 h de vida comienza a presentar episodios de estridor inspiratorio intermitente y atragantamiento.

Ante la sospecha inicial de fístula traqueoesofágica (FTE), se realiza tránsito esófago-gastro-duodenal donde no se observa FTE, únicamente reflujo gastroesofágico. Es valorado por el equipo de Otorrinolaringología (ORL), que realiza rinoscopia y visualiza epiglotis muy malácica y edema de aritenoides. Se decide cambio de fórmula adaptada por hidrolizada y se comienza tratamiento con omeprazol.

Al $6^{\circ}$ día de vida presenta empeoramiento clínico con aumento del trabajo respiratorio en las tomas, estridor e irritabilidad. Como la rinoscopia no es concluyente, se realiza angio-TC cérvico-torácico que objetiva una imagen de densidad quística, redondeada, de $9 \times 10 \times 8 \mathrm{~mm}$ en base de lengua/epiglotis sugestiva de quiste de vallécula (Figura 1). Se inicia tratamiento con dexametasona $(0,15 \mathrm{mg} / \mathrm{kg}$ cada 6 horas) durante 9 días, sin evidente mejoría clínica.

A los 15 días de su nacimiento es trasladada a Unidad de Cuidados Intensivos Neonatales de nuestro hospital. Los hallazgos del Angio-TC son confirmados mediante ecografía, por lo que se programa por el servicio de ORL para cirugía de resección del quiste.

Previo a la cirugía, la paciente presenta trabajo respiratorio manteniendo $\mathrm{SpO}_{2}>94 \%$ con gafas nasales a $4 \mathrm{lpm}$.

Nuestro planteamiento anestésico sobre el manejo de la vía aérea se basa en la intubación orotraqueal en ventilación espontánea, evitando la lesión del quiste por el riesgo de aspiración de su contenido.

Realizamos una monitorización estándar en quirófano con registro de ECG, PANI, $\mathrm{SpO}_{2}$, $\mathrm{EtCO}_{2}$ y temperatura. La inducción anestésica la realizamos con sevofluorano con pauta de concentración descendente $8 \%-6 \%-4 \%$ y una mezcla de aire/ $\mathrm{O}_{2}(60 \%$ y $40 \%)$ para mantener la respiración espontánea del paciente

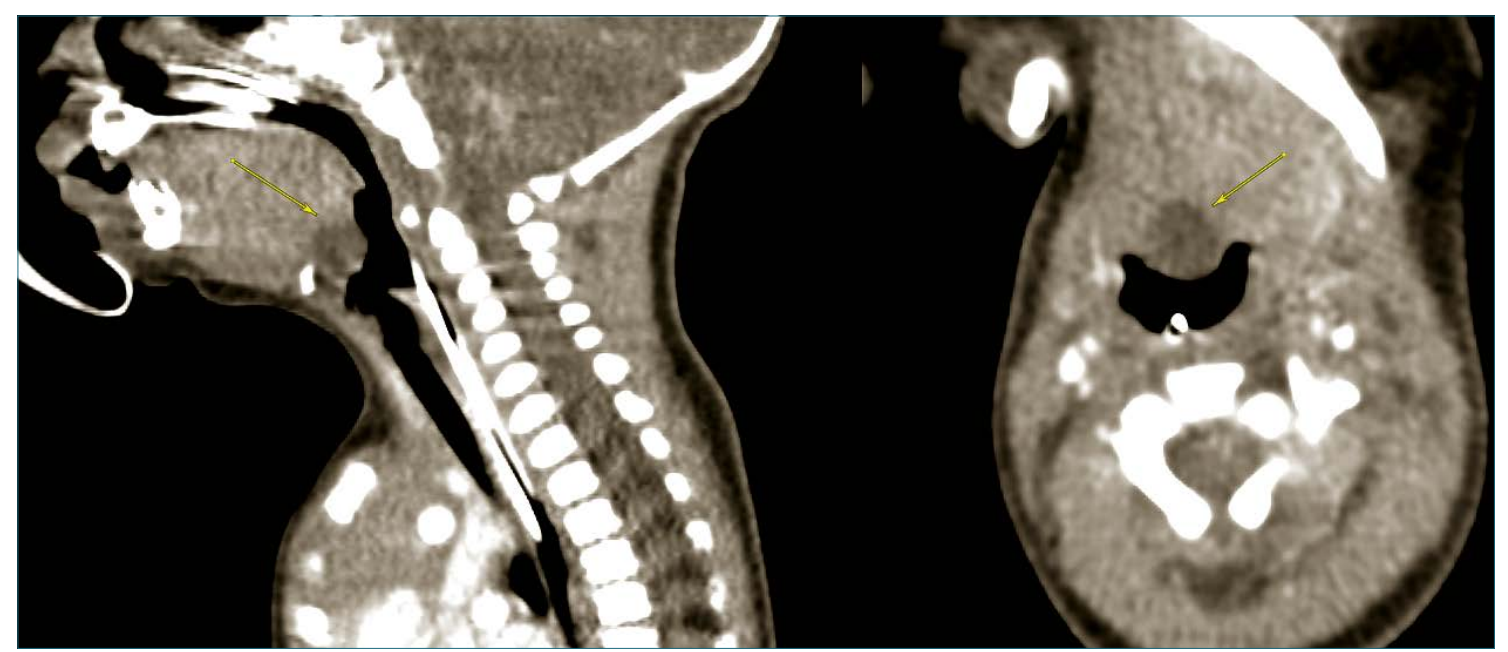

Figura 1. Angio TAC cérvico torácico. *Flechas amarillas: quiste en base lengua/epiglotis $(9 \times 10 \times 8 \mathrm{~mm})$. 
y evitar la apnea. Alcanzado el estadío III plano III de Guedel, fijamos la concentración de Sevofluorano al $2 \%$ y canalizamos vía venosa periférica de $22 \mathrm{G}$ en la mano izquierda y administramos fentanilo a dosis de $1 \mathrm{ug} / \mathrm{kg}$. A continuación, realizamos la topicalización la vía aérea (pilares amigdalinos, supraglotis, epiglotis y glotis) con lidocaína 0,25\% (3 ml) mediante la cánula de atomización.

El equipo de ORL realiza una fibrolaringoscopia flexible previa a la intubación que confirma la presencia del quiste de vallécula desplazando epiglotis sobre vestíbulo laríngeo y glotis.

Realizamos laringoscopia indirecta con videolaringoscopio Glidescope ${ }^{\circledR}$ (Cormack-Lehane I). Esto nos permite ver en todo momento el trayecto de la pala conforme avanzamos. De este modo podemos desplazar el quiste sin riesgo ruptura y visualizar de forma óptima la glotis.

Alcanzada la visualización de la glotis, administramos propofol $2 \mathrm{mg} / \mathrm{kg}$ y rocuronio $0,5 \mathrm{mg} / \mathrm{kg}$ y procedemos a intubación orotraqueal con tubo endotraqueal del $n^{\circ} 3$ con neumotaponamiento (Figura 2a). Realizamos una anestesia general balanceada con sevofluorano para $C A M=1$ y remifentanilo $(0,1-0,3$ $\mathrm{mcg} / \mathrm{kg} / \mathrm{min}$ ).

Durante la cirugía se realiza la exéresis del quiste con extracción y aspiración del contenido de aspecto mucoide (Figura 2b). Se administra dosis de dexametasona $(0,15 \mathrm{mg} / \mathrm{kg})$ y se finaliza la intervención.

La paciente es trasladada a Unidad de Cuidados Intensivos Neonatal (UCIN) intubado y sedoanalgesiado con midazolam $(0,1 \mathrm{mg} / \mathrm{kg})$ y rocuronio $(0,6 \mathrm{mg} /$ $\mathrm{kg}$ ).

A las $3 \mathrm{~h}$ de su llegada a UCIN se retira sedación y se procede a extubación reglada. Tras la extubación del paciente, reinicia clínica de estridor y tiraje inspiratorio que requiere soporte respiratorio con gafas nasales de oxígeno a 5 litros por minuto y pauta con dexametasona. A las $9 \mathrm{~h}$ postextubación, la clínica respiratoria disminuye progresivamente, permitiendo incluso reiniciar nutrición enteral por succión.

A las 48 h de la cirugía, dada la estabilidad clínica la paciente es trasladada a la sala de hospitalización. En la sala de hospitalización, la paciente evoluciona de forma favorable, siendo dada de alta a domicilio sin complicaciones.

\section{Discusión}

El quiste de vallécula es una causa infrecuente de obstrucción de vía aérea superior en niños. Representa un $10,5 \%$ del total de los quistes de laringe[3]. Se

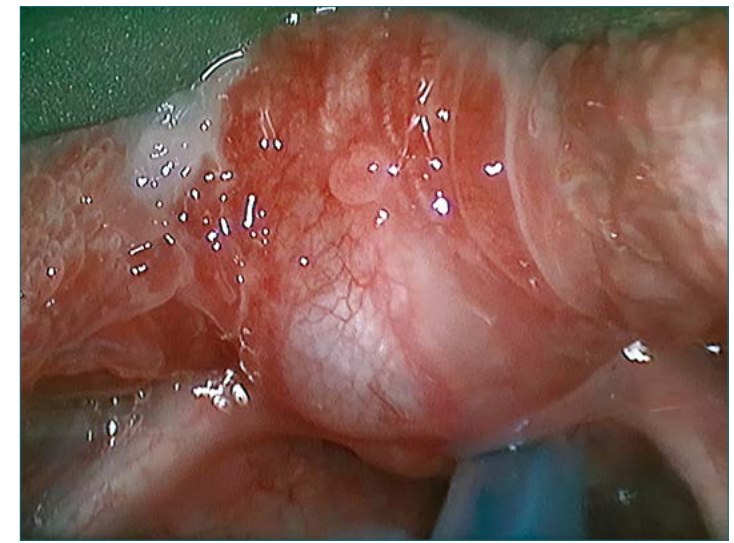

Figura 2a. Quiste de vallécula tras IOT con Glydescope ${ }^{\circledR}$ previa a cirugía.

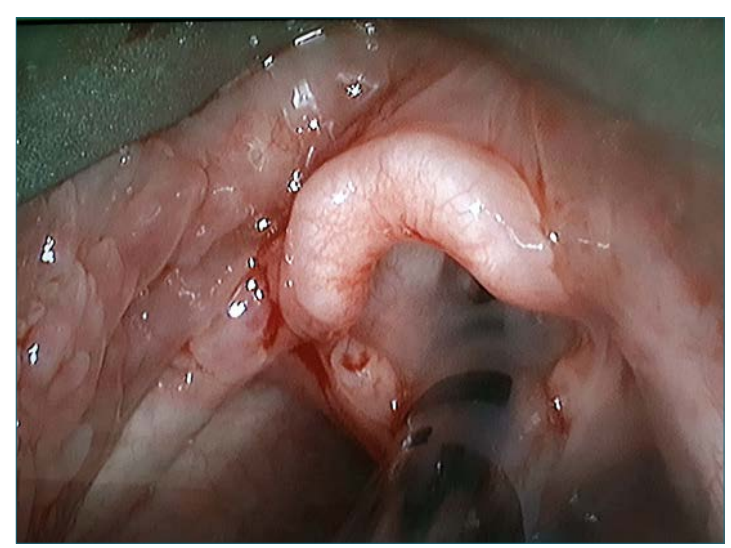

Figura 2b. Epiglotis y glotis tras extracción de material quístico.

originan debido a la obstrucción del drenaje de las glándulas mucosas de la base de la lengua; son, por tanto, quistes de retención mucosa[4]. Los quistes de base de lengua o cara anterior de la epiglotis, están confinados inicialmente a la capa submucosa, pero conforme el quiste incrementa su tamaño, comienza a distorsionar la anatomía de la epiglotis y a ocupar el espacio vallecular.

La obstrucción de la vía aérea por el quiste se debe a dos motivos, tanto por el efecto masa en la hipofaringe, así como por el desplazamiento posterior e inferior de la epiglotis[3].

Una historia clínica detallada reconociendo las posiciones y situaciones que empeoran o mejoran los síntomas obstructivos[2], así como la observación de otros signos clínicos nos pueden hacer sospechar de un quiste congénito laríngeo. En este caso, realizar 
una fibrolaringoscopia flexible con el paciente monitorizado nos puede confirmar el diagnóstico antes de la cirugía[5].

El riesgo de someter a una anestesia general a pacientes programados para resección de quiste de vallécula, se debe fundamentalmente a la potencial pérdida de control de la vía aérea. La obstrucción supraglótica ${ }^{4}$ distorsión de la visión glótica, así como la ruptura del quiste, con la consecuente aspiración de material contenido en él, puede tener consecuencias catastróficas.

Por tanto, el mayor riesgo en estos pacientes es el manejo de la vía aérea. Es recomendable realizar una fibrolaringoscopia previa a la cirugía, para confirmar el diagnóstico y plantear posibles hipótesis de trabajo.

Nosotros consideramos oportuno realizar una intubación en ventilación espontánea con un videolaringoscopio, topicalizando la vía aérea previamente. Esta técnica resultaría de utilidad como alternativa al uso de fibrobroncoscopio en aquellos casos en los que no esté disponible o no se tenga destreza con el mismo. El videolaringoscopio nos permite visualizar en todo momento las estructuras y optimizar los movimientos para disminuir el riesgo de ruptura del quiste; la imagen se proyecta en una pantalla lo que permite la colaboración de otros anestesiólogos durante el procedimiento. Además, su manejo es más sencillo que el fibrobroncoscopio, ya que no requiere tanta destreza.

Existen métodos alternativos al uso de videolaringoscopio. También podemos considerarlos como segunda opción si nuestra técnica inicial falla. Entre ellos tenemos:

- Laringoscopia con pala de Miller (pala recta): desplazar con la misma pala el quiste, aunque conlleva riesgo de atrapamiento y ruptura del quiste.

- Laringoscopio convencional con ayuda de una guía: del mismo modo se puede producir la lesión del quiste.

- Dispositivo supraglótico que permita la intubación con fibrobroncoscopio. Con esta técnica se puede producir una obstrucción a la ventilación.

- En caso de disponer de fibrobroncoscopio, lo indicado es realizar la IOT en ventilación espon- tánea[3]. La dificultad en este caso radica en la disponibilidad de medios (fibrobroncoscopio adecuado al tamaño del tubo endotraqueal). Además, se requiere mayor habilidad para llevar a cabo una IOT en ventilación espontánea en pacientes pediátricos.

Verma et $a^{6}$ describen un caso realizado mediante punción y aspiración del quiste con posterior intubación mediante laringoscopia directa y guía. Nosotros evitamos realizarlo por las complicaciones que pueden derivarse de esta técnica, como la aspiración pulmonar del contenido. Además, el quiste disminuye de tamaño y esto dificulta su localización, por lo que el manejo quirúrgico puede no ser adecuado y predisponer a posteriores recidivas[7].

En el caso clínico descrito por Kariya et al.[8], resuelven la vía aérea con IOT mediante laringoscopia directa convencional y el paciente en ventilación espontánea con la cabeza ladeada hacia el lado donde presenta el quiste, para evitar que ocluya la glotis e impida la ventilación y la intubación.

En nuestro caso contamos con un equipo de cirujanos ORL preparados para realizar una cricotirotomía de emergencia. Es importante tener en cuenta que cuando los planes de abordaje de la vía aérea fallan, el último recurso será la vía aérea quirúrgica.

\section{Conclusiones}

El quiste vallecular en neonatos y niños supone un reto para el anestesiólogo. Es importante ser consciente del potencial peligro que supone para la vía aérea y actuar en consecuencia, disponiendo siempre de diferentes opciones. Además, es fundamental disponer de planes alternativos de rescate para el manejo de la vía aérea en caso de que nuestro plan inicial falle, o el paciente se deteriore.

Por tanto, creemos necesario conocer la lesión mediante TAC y fibrolaringoscopio, y planificar correctamente el manejo anestésico, así como disponer de planes alternativos ante eventuales complicaciones.

\section{Referencias}

1. Namshijar VN, Duckle NV, Sukhthanker DS. Anesthetic management of Vallecular cyst excision in an infant: An airway challenge. Saudi Journal of Anesthesia.

2. Cheng KS, Ng JM, Li HY, Hartigan PM. Vallecular cyst and laryngomalacia in infants: report of six cases and airway management. Anesth Analg. 2002 Nov;95(5):1248-50. https:// doi.org/10.1097/00000539- 
200211000-00026

PMID:12401604

3. Choi YW, Chon JY, Moon HS, Kim JY, Lee JY. Anesthetic management of a neonate with congenital laryngeal cyst. Korean J Anesthesiol. 2012 Sep;63(3):282-3. https://doi. org/10.4097/kjae.2012.63.3.282 PMID:23060991

4. ZC Anton, Gheorghe DC. Vallecular cyst in clinical practice: report of two cases. Journal of Medicine and Life, 2016 (9-3):
288-290.

5. Lee WS, Tsai CS, Lin CH, Lee CC, Hsu HT. Airway obstruction caused by a congenital epiglottic cyst. Int J Pediatr Otorhinolaryngol. 2000 Jul;53(3):22933. https://doi.org/10.1016/ S0165-5876(00)82012-1 PMID:10930640

6. Verma RK, Panda NK, Bhatia N. Anesthetic and Surgical Considerations in Giant Vallecular Cyst with coexistent Laryngomalacia of neonate. Aust J Otolaryngol.
2015;2(7):1054.

7. Hsieh WS et al. Vallecular cyst: an uncommon cause of stridor in newborn infants. Eur J Pediatr. 2000; 159 (1.2):79-81. https:// doi.org/10.1007/PL00013809.

8. Kariya N, Nishi S, Funao T, Nishikawa K, Asada A. Anesthesia induction for a difficult intubation infant with a laryngeal cyst. J Clin Anesth. 2003 Nov; 15(7):534-6. https:// doi.org/10.1016/j.jclinane.2003.01.002 